\title{
François Péron et la question de la civilisation aux antipodes
}

Francois Péron and the Question of Civilization in the Antipodes

Jean-Luc Chappey

\section{(2) OpenEdition \\ 1 Journals}

Édition électronique

URL : https://journals.openedition.org/ahrf/13079

DOI : $10.4000 /$ ahrf.13079

ISSN : 1952-403X

Éditeur :

Armand Colin, Société des études robespierristes

Édition imprimée

Date de publication : 1 mars 2014

Pagination : 139-159

ISBN : 978-2-200-9083-2790-8

ISSN : 0003-4436

Référence électronique

Jean-Luc Chappey, «François Péron et la question de la civilisation aux antipodes », Annales

historiques de la Révolution française [En ligne], 375 | janvier-mars 2014, mis en ligne le 01 juillet 2017, consulté le 05 juillet 2021. URL : http://journals.openedition.org/ahrf/13079 ; DOI : https://doi.org/ 10.4000/ahrf. 13079 


\title{
FRANÇOIS PÉRON ET LA QUESTION DE LA CIVILISATION AUX ANTIPODES
}

Jean-Luc CHAPPEY

\begin{abstract}
Membre de l'expédition maritime du capitaine Nicolas Baudin vers les terres australes (1800-1804), François Péron (1775-1810) rédige entre 1804 et 1809 une série de rapports, récits et descriptions sur les diverses populations, sauvages et européennes, qu'il a pu observer durant son voyage. Ces différentes productions ont, depuis longtemps, suscité l'intérêt des historiens, particulièrement des historiens de l'anthropologie. Considérées dans la perspective des instructions de voyage rédigées par les membres de la Société des Observateurs de l'homme, les observations de François Péron marqueraient une rupture, symbolisée par l'usage du dynamomètre et de la promotion de la mesure de la force musculaire, dans la vision des populations sauvages dont la faiblesse physique « naturelle » rendrait impossible l'accès au processus de progrès et de perfectibilité. Ses observations anthropologiques constitueraient ainsi la fin de la vision du « bon sauvage » portée par l'héritage des Lumières. Dans cette perspective, Péron apparaît encore comme le représentant, et l'acteur, d'une rupture dans la conception d'une humanité commune, les populations sauvages étant réduites à un statut d'irréductible étrangeté. Or l'anthropologie de Péron s'inscrit dans le mouvement général de remise en ordre politique et sociale qui caractérise l'Empire, les modalités particulières de la description des populations australes participant à la mise l'écart des idéaux politiques de la Révolution française et plus précisément encore, du projet républicain de la République directoriale.
\end{abstract}

Mots-clés : voyages, race, anthropologie, histoire, civilisation

Membre de l'expédition maritime du capitaine Nicolas Baudin vers l'Australie, François Péron (1775-1810) occupe une position particulière 
dans l'histoire de l'anthropologie française ${ }^{1}$. Au cours des dernières décennies du XIX ${ }^{\mathrm{e}}$ siècle, il a été érigé, par les partisans de l'anthropologie physique réunis autour de Paul Broca au sein de la Société d'anthropologie de Paris, en promoteur des nouvelles règles d'étude de l'homme et d'analyse des sociétés : ses observations sur les populations des terres australes (Nouvelle Zélande, Van Diémen, Nouvelle-Galles du Sud et Timor) rapportées de son voyage et fondées sur la mesure de la force musculaire auraient en effet permis de classer et de hiérarchiser les diverses races humaines selon des principes «naturels $»^{2}$, jouant ainsi un rôle essentiel dans la construction de la «République raciale $»^{3}$. Aujourd'hui encore, les historiens français et anglo-saxons de l'anthropologie le considèrent comme l'acteur majeur, sinon central, d'un renversement des fondements épistémologiques et méthodologiques de l'anthropologie au tournant des $\mathrm{XVIII}^{\mathrm{e}}$ et $\mathrm{XIX}^{\mathrm{e}}$ siècle : son regard désenchanté, voire critique, sur les populations « sauvages » aurait en effet signé la fin d'un certain idéal des Lumières et l'entrée dans une nouvelle ère d'affirmation de la supériorité blanche et européenne ${ }^{4}$. La publication du Voyage de découverte en $1807^{5}$ marquerait ainsi une « rupture, un renversement des perspectives dans l'ordre des représentations du sauvage qui, bien que toujours perçu comme

(1) L'expédition Baudin est l'objet de récents et enrichissants renouvellements historiographiques. Cette impulsion a été largement assurée par les travaux menés par des chercheurs australiens dont les recherches ont été particulièrement dynamiques ces dernières années. Aux côtés des études portant spécialement sur l'implantation des Anglais en 1788 que le Bicentenaire a largement stimulés (Cf. par exemple, Inga Clendinnen, Dancing with Strangers, Melbourne, Texte Publishing, 2003), on se tournera plus précisément vers Jean ForNASIERO, Peter MONTEATH and John WESTSoOBy, Encountering Terra Australis. The Australian Voyages of Matthew Flinders, Wakefield Press, 2010 (réed.) ; Shino KonISHI, «François Péron and the Tasmanians : an unrequited romance », http://epress.anu.edu.au/aborig_history/transgressions/mobile_devices/ch01.html. On se reportera particulièrement aux ressources mises à disposition sur le site « The Baudin Legacy Project » hébergé à l'Université de Sydney : http://sydney.edu.au/arts/research/baudin/project/index.shtml. Voir également au sujet des débats - et polémiques - suscités autour du Bicentenaire de la découverte de l'Australie : Stuart JANSON and Stuart MACINTYRE (ed.), Making the Bicentenary, Melbourne, Melbourne University Press, 1988 ; (en français) : Isabelle MERLE, «Le Mabo Case. L'Australie face à son passé colonial », Annales HSS, 1998, 2, p. 209-229.

(2) Mondher KILANI, L'invention de l'autre, Lausanne, Payot, 1994 ; Claude BLANCKAERT, «Code de la nature et loi de l'histoire. Les appropriations naturalistes du "primitif contemporain" entre $\mathrm{XVIII}^{\mathrm{e}}$ et XIX ${ }^{\mathrm{e}}$ siècles », Gabrielle BAGLIONE et Claude BlANCKAERT (dir.), L'Autre. Les Naturels vus par l'Occident, Le Havre, Éditions du Muséum d'histoire naturelle du Havre, 2008, p. 10-15.

(3) Carole REYNAUD-PALIGOT, La République raciale. Paradigme racial et idéologie républicaine (1860-1930), Paris, PUF, 2006.

(4) Sur le destin de Péron dans l'historiographie, voir la très riche mise au point de Jean FORNASIERO, « Deux observateurs de l'homme aux antipodes. Nicolas Baudin et François Péron », Michel JANGOUX (dir.), Portés par l'air du temps : les voyages du capitaine Baudin. Études sur le XVIII ${ }^{e}$ siècle, Bruxelles, Université de Bruxelles, 2010, p. 158-170.

(5) François PÉRON, Voyage de découvertes aux terres australes exécuté par ordre de sa majesté l'Empereur et Roi sur les corvettes Le Géographe, le Naturaliste, Paris, Imprimerie impériale, 1807 , tome 1 . On pourra se reporter à la réédition récente du fac-similé par le CTHS en 2011. 
un primitif, comme un homme de la nature, n'est plus ici valorisé par cette qualité et cette appartenance, n'est plus gratifié d'une perfection ou d'une puissance physiques que l'on voulait déduire de celle-là. Péron soutient au contraire que la trop grande proximité du « sauvage » avec la nature, affaiblit son corps et altère son esprit $»^{6}$. Dans cette optique, Péron apparaît sous les traits d'un renégat doublé d'un mauvais savant ${ }^{7}$. Sans qu'il soit ici question de laver sa réputation des accusations portées contre lui, il s'agit d'interroger les conditions et les enjeux du basculement de son regard porté sur les populations et les sociétés «sauvages » en tentant de réinscrire la publication de son Voyage dans les dynamiques politiques et les mutations profondes qui caractérisent l'espace intellectuel entre le Consulat et l'Empire.

\section{Publication et réputation savante}

En octobre 1800, deux navires, le Géographe et le Naturaliste, respectivement commandés par Nicolas Baudin et Emmanuel Hamelin, quittent Le Havre à destination des terres australes ${ }^{8}$. Pour Joseph-Marie de Gérando, qui a participé à la rédaction d'une partie des instructions à destination de l'équipage, cette expédition est autant un voyage dans le temps que dans l'espace : il s'agit en effet de partir à la recherche des populations primitives susceptibles de mieux comprendre les différents stades des progrès de la civilisation. À bord des navires sont présents vingt-deux savants, réunis parfois difficilement par les autorités. Aux côtés des naturalistes, des médecins ou des astronomes chargés de collecter les matériaux les plus divers, prend place François Péron, jeune médecin monté à bord comme « anthropologiste », spécialement chargé d'observer les populations. On sait que cette expédition, quoique planifiée depuis plusieurs années et dirigée par des personnalités reconnues et compétentes, se révèle particulièrement éprouvante : dès les premiers mois, aux conflits qui divisent les membres de l'équipage, s'ajoutent des conditions de vie particulièrement difficiles qui rendent compte des désertions et des décès qui rythment le voyage. Bien qu'elle suscite au départ l'intérêt des savants,

(6) Jean Copans et Jean JAmin, Aux origines de l'anthropologie française. Les mémoires de la Société des observateurs de l'homme en l'an VIII, Paris, Le Sycomore, 1978, p. 32.

(7) Miranda HugHES, « Philosophical Travelers at the End of the Earth: Baudin, Péron, and the Tasmanians », Roderick WEIR HoME (ed.), Australian Science in the Making, Cambridge, Cambridge University Press, 1988, p. 23-44.

(8) Michel JangouX, Portés par l'air du temps: les voyages du capitaine Baudin, op. cit. ; Bertrand DAUGERON, À la recherche de l'espérance. Revisiter la rencontre des aborigènes tasmaniens avec les Français (1772-1802), Dacres Éditions, 2013. 
voire d'un large public, l'expédition maritime cesse de figurer dans les colonnes des journaux qui signalent néanmoins la mort du capitaine Baudin en 1803. Le retour des rescapés l'année suivante attire de nouveau l'attention des milieux savants, particulièrement des naturalistes du Muséum national d'histoire naturelle, destinataires privilégiés des collections importantes rapportées par les voyageurs. Bien que le genre ait connu un essor quantitatif important, la publication d'un récit de voyage reste un enjeu majeur pour celui qui cherche à construire sa réputation auprès des savants et du public. Ainsi, Jean-Baptiste Bory de Saint-Vincent, qui a quitté l'expédition rapidement, n'hésite pourtant pas à prendre de l'avance en publiant dès 1804 un premier récit de l'expédition ${ }^{9}$. Pour ces jeunes savants qui avaient choisi de quitter Paris et la proximité avec les «patrons » des grandes institutions scientifiques, il s'agit dès lors de tirer profit du voyage, une quête de reconnaissance qui donne lieu à une vive concurrence, d'autant que l'espace politique et intellectuel a subi de profondes transformations depuis leur départ en 1800.

D'un côté, les principales institutions savantes qui avaient été à l'origine de l'expédition n'existent plus ou ont été réorganisées : l'Institut national subit en janvier 1803 une transformation majeure marquée par la suppression de la Classe des sciences morales et politiques ; par ailleurs, la Société des Observateurs de l'homme s'éteint progressivement à la fin de l'année 1803. Ces transformations institutionnelles sont liées à de profondes mutations de l'ordre des savoirs, manifestées par l'abandon de l'idéal encyclopédique (illustré par la disparition de l'Idéologie et de l'anthropologie des Observateurs de l'homme) et par la promotion de nouvelles formes de spécialisation ${ }^{10}$. De l'autre, ces changements sont indissociablement liés aux ruptures qui caractérisent l'espace politique, la première d'entre elles étant l'abandon progressif de l'idéal républicain

(9) Voyage dans les quatre principales îles des mers d'Afrique, fait par ordre du gouvernement, pendant les années neuf et dix de la république (1801 et 1802), avec l'Histoire de la traversée du capitaine Baudin jusqu'au Port-Louis de l'île Maurice, Paris, F. Buisson, an XIII (1804). Sur Bory de Saint-Vincent, cf. Hervé FERRIÈRE, Bory de Saint-Vincent. Évolution d'un naturaliste voyageur, Syllepse, 2009.

(10) Je renvoie sur ces mutations à mes travaux, en particulier « Les enjeux d'une anthropologie dans l'ordre des savoirs autour de 1800. Retour sur la Société des Observateurs de l'homme », dans Anja BANDAU, Marcel DORIGNY et Rebekka von MALLINKRODT (dir.), Les mondes coloniaux à Paris au XVIII siècle. Circulation et enchevêtrement des savoirs, Karthala, p. 97-120 ; « De la science de l'homme aux sciences humaines : enjeux politiques d'une configuration de savoir (1770-1808) », Revue d'histoire des sciences humaines, $\mathrm{n}^{\circ} 15, \mathrm{p}$. 43-68. Voir aussi Bernard GAINOT, «Les Lettres philosophiques de Jacques-Rigomer Bazin, publiées en 1807. Un héritage de la Décade philosophique », Jacques BERNET et Emmanuel CHERRIET (dir.), 1807 : Apogée de l'Empire, Valenciennes, PU de Valenciennes, 2009, p. 275-299. 
du Directoire qui pouvait encore perdurer lors des premières années du Consulat. Dès 1802, la décision de rétablir l'esclavage ruine les fondements de la vision coloniale et civilisatrice qui justifiaient, aux yeux des républicains, l'idée de considérer les membres des populations éloignées et « sauvages » comme des frères. On sait que, dès son arrivée au pouvoir, Bonaparte et le ministre de la Marine Forfait travaillent au rétablissement du contrôle de la métropole sur des territoires coloniaux qui ont su s'affranchir de la domination de la France. Cette politique qui justifie le rétablissement de l'esclavage suscite des résistances importantes qui aboutissent, l'année même du retour de l'expédition, à la proclamation de l'indépendance d'Haïti. Le réel traumatisme que cause cet événement en France n'en justifie que plus les aberrations racistes publiées par des esclavagistes qui, dès les premières années du Consulat, retrouvent une indéniable audience. De manière plus modérée, mais de façon tout aussi efficace, le jeune JulienJoseph Virey assure la diffusion des thèses polygénistes qui, sans disparaître complètement, avaient pourtant été mises sous silence durant la République directoriale. Dès lors, on le voit : les conditions intellectuelles et politiques qui avaient constitué le contexte de préparation et de départ de l'expédition n'ont pas seulement disparu ; elles se sont inversées. Par conséquent, les acteurs doivent s'adapter et trouver les ressources susceptibles de répondre aux nouvelles attentes des autorités politiques et scientifiques.

Dès son retour en France, Péron fait l'objet de nombreux éloges de la part du monde savant, en particulier de la part de Georges Cuvier, personnalité «montante » au sein des naturalistes parisiens. Ancien élève du médecin Jean-Noël Hallé, protégé par les naturalistes Étienne-BernardGermain Lacépède et Antoine-Laurent Jussieu à son départ en 1800, Péron change ainsi opportunément de patron à son retour, un changement qui illustre les mutations des rapports de force au sein de l'espace scientifique. Professeur au Muséum national d'histoire naturelle, membre de la première Classe de l'Institut national, Cuvier parvient en effet à attirer le jeune Péron qui trouve dans ce patronage un moyen de s'ouvrir les portes des institutions consacrées et de trouver les ressources nécessaires à la vie parisienne. Pour Cuvier, naturaliste « sédentaire» soucieux de renforcer son pouvoir au sein du Muséum, les collections naturalistes dont Péron était responsable et les dessins de Charles Alexandre Lesueur, constituent des matériaux particulièrement précieux. Anthropologiste en 1800, Péron s'affirme ainsi progressivement comme zoologiste, un domaine qu'il investit dès 1804 
en présentant à l'Institut, sous la tutelle de Cuvier, plusieurs mémoires ${ }^{11}$. En février 1805, ces efforts sont récompensés par son élection comme membre correspondant de la section d'anatomie et zoologie de la première Classe de l'Institut national. Péron n'a pourtant pas totalement abandonné l'observation de l'homme et des sociétés : en 1804, il publie en effet un article sur les femmes hottentotes. La publication en 1807, sur les presses de l'Imprimerie impériale, de son Voyage lui permet non seulement de mettre au jour les nombreux matériaux qu'il a réunis sur ce sujet, mais aussi d'imposer le récit quasi-officiel de l'expédition ${ }^{12}$.

\section{Péron et les populations sauvages}

Sous l'Empire, publier un récit de voyage consiste à s'inscrire dans un genre littéraire particulièrement en vogue. Victime de son succès commercial, ce genre est même l'objet de nombreuses critiques de la part des membres de la communauté savante qui, depuis les dernières décennies du XVIII ${ }^{\mathrm{e}}$ siècle, en critiquent les règles. Dans les instructions qu'il rédige en 1800 pour les membres de l'expédition Baudin, Cuvier en venait même à mettre en cause l'utilité de tels témoignages, insistant plutôt sur l'importance scientifique des collectes d'objets et de matériaux. Ces récits demeurent pourtant nécessaires autant pour les connaissances qu'ils fournissent sur les modes de vie des populations qui ne sauraient se réduire à l'analyse de collections d'objets que pour la réputation du voyageur auprès d'une administration susceptible de lui fournir des ressources nécessaires à la poursuite de sa carrière et d'un public de lecteurs plus large. Dans son récit, Péron n'hésite d'ailleurs pas à utiliser les stratégies rhétoriques classiques (héroïsation du narrateur, valorisation de l'anecdote...) pour

(11) François PÉRON, « "Mémoire sur quelques faits zoologiques applicables à la théorie du globe" lu à la Classe des sciences physiques et mathématiques de l'Institut national, le 30 vendémiaire an XIII [22 oct. 1804] », Journal de physique, de chimie, d'histoire naturelle et des arts, 1804, vol. 59, p. 463-479 ; « Mémoire sur le nouveau genre Pyrosoma », Annales du Muséum national d'Histoire naturelle, tome 4, an XII [1804], p. 437- 446 ; « Notice d'un mémoire sur les animaux observés pendant la traversée de Timor au Cap Sud de la Terre de Van Diemen », Bulletin des sciences de la Société philomatique, $\mathrm{n}^{\circ}$ XI, $8^{\mathrm{e}}$ année, tome III, $\mathrm{n}^{\circ}$ 95, pluviôse an 13 [décembre 1804-janvier 1805], p. 269-270 ; «Observations sur la dysenterie des pays chauds et sur l'usage du bétel », Journal de physique, de chimie, d'histoire naturelle et des arts, vol. 59, 1804, p. 290-299.

(12) Bronwen Douglas, «Seaborne Ethnography and the Natural History of Man », The Journal of Pacific History, vol. 38, n¹, 2003, p. 3-27 ; Id., « Slippery word, ambigous praxis : "Race" and late-XVIIIth century voyagers in Oceania », The Journal of Pacific History, vol. 41, $\mathrm{n}^{\circ} 1,2006, \mathrm{p}$. $1-29$. 
piquer la curiosité de son lectorat particulièrement attiré par des terres australes encore méconnues ${ }^{13}$.

À travers son récit, il cherche encore à renforcer sa propre légitimité de savant, une position qui détermine assurément les sévères critiques qu'il porte contre le capitaine Baudin érigé autant en personnalité tyrannique qu'en figure de voyageur-philosophe du XVIII ${ }^{\mathrm{e}}$ siècle contre laquelle Péron, voyageur «moderne » symbolisant l'entrée dans le XIX ${ }^{\mathrm{e}}$ siècle, tente de se construire $^{14}$. Mettant en valeur son travail d'enquêteur, ce dernier assume en effet une position de savant-collecteur dont les observations sont appuyées sur des «mesures» qui doivent les rendre exactes. Ces mesures sont réalisées grâce au dynamomètre dont la présentation des résultats constitue l'objet du chapitre XX qui clôt l'ensemble du récit ${ }^{15}$. Pour bien lire ce récit de voyage, il conviendrait donc de commencer par ces tableaux qui font la synthèse des différentes expériences menées pour comparer la force musculaire des diverses populations sauvages et civilisées : ce sont en effet ces tableaux et les conclusions qui en sont issues concernant la hiérarchie des différentes sociétés humaines qui donnent une cohésion à l'ensemble du récit. De ces expériences réalisées sur les divers individus des différentes sociétés il en ressort une hiérarchie : "1/Que les habitants de la terre de Diémen, les plus sauvages de tous, les enfants de la nature par excellence, sont les plus faibles; 2/Que ceux de la Nouvelle Hollande, qui ne sont guère plus civilisés, sont plus faibles que les habitants de Timor ; 3/Que ces derniers à leur tour sont beaucoup plus faibles, soit des reins, soit des mains, que les Anglais et les François. Nous pouvons donc déduire de l'ensemble de ces résultats la conséquence suivante : le développement de la force

(13) Les récits sur les terres australes sont encore rares à l'époque. Les journaux du premier voyage de James Cook (1768-1770) - auquel participe James Banks - sont immédiatement traduits en français en 1772, 1778 et 1782. On sait que le Mayençais Georg Forster se forge également une réputation en publiant un récit de voyage vers les terres australes. Avant eux, pensons également à l'Histoire des navigations aux terres australes de De Brosses (1756). Parmi les très nombreux travaux consacrés à l'intérêt pour le Pacifique et les terres australes, cf. Nicholas THOMAS, The Extraordinary Voyages of Captain James Cook, New York, Walker Compagny, 2003 ; Kapil RAJ, «18th-Century Pacific Voyages of Discovery. "Big Science" and the Shaping of an European Scientific and Technological Culture », History and Technology, 2000, vol. 17, p. 79-98.

(14) Jean-Paul FAIVRE, L'expansion française dans le Pacifique de 1800 à 1842, Paris, Nouvelles éditions latines, 1953 ; Frank HORNER, The French Reconnaissance : Baudin in Australia (1801-1803), Carlton, Melbourne University Press, 1987 ; Margaret SANKEY, «F. A. Péron et l'écriture de la science », Cahiers de sociologie économique et culturelle, 1988, vol. 9, p. 37-46 ; Jean-Luc CHAPPEY, « Le capitaine Baudin et la Société des observateurs de l'homme. Questions autour d'une mauvaise réputation », dans Michel JANGOUX (dir.), op. cit., p. 145-155.

(15) Notons que Péron abandonne son instrument à l'Isle-de-France : « Je l'ai remis, avec l'autorisation du Gouverneur de la colonie, à M. Chapotin, médecin du Gouvernement et j'ai lieu d'espérer qu'entre ses mains il pourra fournir un jour les résultats les plus précieux », François PÉRON, Voyage, op. cit., p. 457. 
physique n'est pas toujours en raison directe du défaut de civilisation ; il n'est pas un produit constant, il n'est pas un résultat nécessaire de l'état sauvage $»^{16}$.

Le fait que Péron ait embarqué le dynamomètre dès son départ ne laisse pourtant en rien présager de son usage postérieur dans la construction du récit. Promu par son concepteur Edme Régnier (1751-1825) en 1798 pour mesurer la force musculaire des hommes et des animaux ${ }^{17}$, l'instrument dont le physicien Charles-Augustin Coulomb (1736-1806) valorise l'utilité dans les travaux scientifiques, est officiellement adopté par les autorités en 1800 qui en ordonnent l'usage dans les écoles d'artillerie. À cette date, le dynamomètre sert également à l'instruction des jeunes médecins, Péron ayant pu être ainsi familiarisé à l'usage de cet appareil ${ }^{18}$.

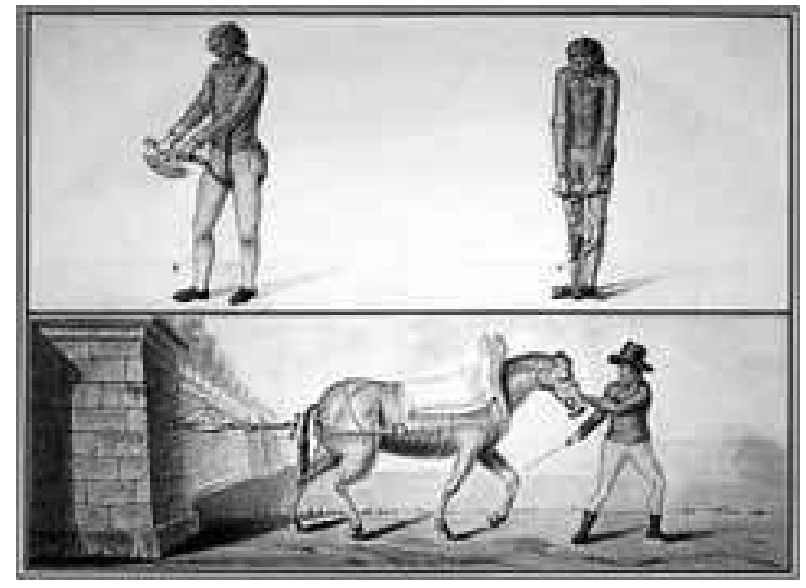

Figure 1. (C) Musée des arts et métiers/J.-M. Courant/Dephti-Ouest Aquarelle exécutée pour le Conservatoire par Dronord (paraphe). Papier vergé [46,5 x 63]. Vers 1798.

Que Péron ait effectivement réalisé des expériences et des mesures sur la force musculaire des divers membres des sociétés européennes ou aborigènes rencontrées est une chose. Qu'il ait choisi de présenter ces mesures comme le critère permettant, d'un côté, de marquer une nette distinction entre les populations civilisées et les populations sauvages,

(16) Ibid., p. 457.

(17) Edme Régnier présente son invention dans le Journal de l'École polytechnique, (t. II, prairial an VI [1798], p. 160-172) puis dans une brochure publiée la même année.

(18) Une brochure de floréal an VIII [avril-mai 1800] indique que «plusieurs écoles de médecine s'en servent maintenant pour l'instruction des élèves », Description et usage de trois sortes de briquets défensifs à pistolet... par le C. Regnier,... avec une Notice sur les inventions de l'auteur, Paris, impr. de la République, an VIII. 
de l'autre, de hiérarchiser ces populations sauvages entre elles, elève d'une autre démarche : il s'agit finalement de réduire toute la démarche proprement anthropologique construite, lors du voyage, sur de nombreuses observations aux seules mesures présentées dans les différents tableaux publiés en fin de volume. Ces mesures déterminent ainsi largement la lecture de l'ouvrage dans son ensemble et renforcent encore le caractère particulièrement négatif des jugements portés par l'auteur sur les diverses populations. Les nombreux détails souvent anecdotiques donnés sur les habitudes et les modes de vie des populations locales apparaissent comme autant de matériaux qui sont finalement validés par ces mesures dont le récit constitue, à l'inverse, non seulement une illustration, mais une explication. Par son usage des tableaux, Péron marque indéniablement une rupture avec la forme narrative des récits de voyage : en utilisant ce mode de représentation visuelle des différences entre les populations observées, il s'agit d'imposer une nouvelle forme de validité qui participe à la légitimation d'un nouveau langage scientifique, celui des chiffres et des observations fondées sur l'usage d'un instrument. La lecture des tableaux montre pourtant que l'objectivité et la neutralité des expériences réalisées avec le dynamomètre et des conclusions qui en découlent sont loin d'être garanties : non seulement Péron n'indique en rien la manière dont ont été réalisées ses mesures, mais les commentaires négatifs qu'il porte sur différents indigènes dont la force musculaire a été mesurée montrent que les mesures ne sont là que pour renforcer davantage la coupure naturelle qu'il établit entre « sauvages » et « civilisés ».

C'est par la description des modes de vie des populations, de l'organisation des diverses sociétés, que Péron cherche à rendre compte et à justifier les différences de force musculaire. Selon lui, les populations les plus « faibles » sont aussi celles qui vivent dans l'état le plus sauvage, état qui ne saurait être rapporté aux conditions naturelles offertes par un milieu qui serait hostile : en effet, dans le cas du Timor, c'est parce les conditions naturelles sont les plus favorables et offrent aux populations des ressources abondantes que ces populations, ne connaissant aucune contrainte et, n'étant pas obligés de travailler ni de faire aucune espèce d'effort, ne peuvent développer leur force physique. Paresse et « léthargie » sont ainsi les produits d'un milieu trop favorable :

«Malheureusement cette facilité prodigieuse de satisfaire à tous les besoins de la vie, cette abondance de tous les biens sans mélange de peines et de labeurs, ont déterminé dans toute la nation un caractère d'apathie et d'indifférence si décidé, une aversion si forte pour le travail et la fatigue, 
que l'idée seule de s'y livrer attristerait un Malais de ces régions. Demeurer accroupi une partie de la nuit et du jour, le derrière sur les talons, à l'ombre d'un tamarinier, d'un palmier ou d'un manguier ; mâcher sans cesse le betel, boire du calou, faire trois ou quatre repas assez légers [...] tresser quelques nattes, ou s'occuper d'autres ouvrages aussi légers, aussi faciles ; se baigner enfin, se peigner, se frictionner avec de l'huile de coco : tel est le cercle invariable des occupations d'un Malais libre à Timor. Pour les esclaves, ils sont en si grand nombre dans chaque maison, on exige si peu de travaux, ils les exécutent si lentement, que leur existence particulière n'est guère moins oisive que celle de leurs maîtres $»^{19}$.

Plus faibles encore que celles du Timor, les populations du Dièmen et de la Nouvelle Hollande, face à un environnement naturel particulièrement hostile et à un état de désorganisation politique et sociale, sont, à l'inverse, accablées par la «misère $»^{20}$. Dans les dessins de Petit et Lesueur, les représentations particulièrement longilignes des bras et des jambes des indigènes mettent encore en évidence l'absence, à des degrés divers, de force musculaire ${ }^{21}$. Selon les fondements théoriques issus des fameux « rapports du physique et du moral », cette faiblesse physique, désormais établie par des mesures exactes, renvoie à une faiblesse morale qui se marque autant par le caractère souvent «brutal », « féroce », « perfide » ou « grossier », que le récit de rencontres parfois tumultueuses ne fait qu'illustrer, que par l'absence de marque ou d'expression de sentiments qui seraient présentes naturellement chez les populations européennes ${ }^{22}$. Péron montre son étonnement face à ce qu'il estime être l'absence d'émotions chez les «sauvages » qui ignorent, selon lui, le rôle des baisers et des caresses dans les échanges entre les individus. Dès lors, seules la méfiance et la prévention doivent accompagner la rencontre : « nous en tirions la conséquence, qu'il

(19) François PÉRON, Voyage, op. cit., p. 460-461.

(20) Ibidem, p. 466: «mais cette disette d'aliments, cette nécessité de faire usage des substances les plus dégoûtantes, ces fatigues excessives pour se les procurer, ne peuvent-elles pas être considérées à leur tour comme un résultat immédiat et nécessaire de l'état sauvage dans lequel ces peuplades malheureuses végètent encore ! ».

(21) Anne LAFONT, « The Visual Terms of Cultural Encounters : Petit and Cuvier Australian Experiment », dans Sue Ann PRINCE (dir.), Of elephants and Roses. Encounters with French Natural History, 1790-1830, Philadelphie, American Philosophical Society, 2013, chapitre 13, p. 157-167; Anne LAFONT (dir.), «Étrange étrangeté : la science au cœur de la représentation de l'Africain », L'artiste savant à la conquête du monde moderne, Strasbourg, Presses Universitaires de Strasbourg, 2010, p. 141-156.

(22) Dans ses « Observations sur le tablier des femmes hottentotes » (1805), Péron distingue les Hottentots des Boschimans observés lors d'une escale au Cap de Bonne Espérance. Selon lui, ces hordes rebelles et sauvages, vivant sans structure politique, allient difformités physiques et difformités morales. 
ne faut se présenter devant ces peuples qu'avec des moyens suffisants pour contenir leur mauvaise volonté ou repousser leurs attaques $»^{23}$. On mesure le chemin parcouru par Péron depuis le texte présenté aux autorités administratives et scientifiques en 1799 pour justifier son désir de participer à l'expédition maritime et légitimer l'intérêt à apporter aux populations indigènes. Dans ses «Observations sur l'anthropologie... », Péron est particulièrement critique contre les effets des «progrès » de la civilisation. Il dénonce la « cohorte effroyable de maladies de toute espèce qui n'ont d'autre source que les progrès même de notre civilisation $\gg^{24}$ et appelle à se tourner vers le passé et les peuples sauvages pour tenter de trouver les remèdes aux différents maux (surpeuplement urbain...) qui caractérisent les sociétés civilisées : « délivrez, dis-je, l'humanité de toutes ces maladies nécessairement inconnues à des peuples moins civilisés, de combien alors la somme de leurs infirmités est moindre que la nôtre $! »^{25}$.

Entre 1800 et 1807, Péron semble ainsi s'être non seulement converti aux nouvelles normes de l'étude des hommes et des sociétés humaines (étude désormais réduite à des mesures, que ce soit des crânes avec Cuvier ou Gall ou des forces musculaires), mais encore conformé aux nouvelles formes de partage et de division entre les populations « civilisées » et les populations sauvages, ces dernières étant naturalisées comme inéluctablement inférieures. "Rousseauiste exalté en 1800, il revient de son périple quelques années plus tard en enfant du siècle, contempteur des robinsonnades $»^{26}$. Aux côtés d'autres naturalistes et médecins sous l'Empire, Péron semble ainsi contribuer à renforcer encore cette "idéologie » européocentriste sur laquelle peut s'appuyer le nouveau régime pour légitimer davantage la remise en place de la politique esclavagiste ${ }^{27}$. Péron n'est pas seul à participer au renversement du regard porté sur les «sauvages » : Jean-Marc Itard en publiant en 1807, l'année de la suppression de la Décade philosophique, son fameux rapport sur l'enfant sauvage de l'Aveyron témoigne de son échec à l'éduquer et, de fait, rompt avec l'idéal de régénération hérité des Lumières et participe à la naturalisation de la rupture entre état civilisé et état sauvage. Entre Péron et Itard, existerait-il un même mouvement de désenchantement face à la figure du « bon » sauvage, mouvement qui participerait à une forme d'invention du $\mathrm{XIX}^{\mathrm{e}}$ siècle ?

(23) François PERON, Voyage, op. cit., p. 285.

(24) Id., « Observations sur l'anthropologie », op. cit., p. 183.

(25) Ibidem.

(26) Claude BlanCKAERT, «Code de la nature et loi de l'histoire. Les appropriations naturalistes du "primitif contemporain" entre XVIII ${ }^{\mathrm{e}}$ et XIX ${ }^{\mathrm{e}}$ siècles », op. cit., p. 10.

(27) Yves BÉNOT, La démence coloniale sous Napoléon, Paris, La Découverte, 2006. 


\section{Péron et l'histoire des civilisations}

Comme nous l'avons dit précédemment, Péron se révèle, en 1800, très critique face aux produits des progrès de la civilisation. Ce « mal du siècle » semble constituer alors un trait commun chez de nombreux jeunes médecins tels Virey ou encore Jacques-Louis Moreau de la Sarthe. Souvent proches du professeur d'hygiène Hallé, ces représentants de la jeune génération critiquent souvent violemment les effets négatifs des " progrès » - comme l'urbanisation ou l'industrialisation - sur les conditions de vie des populations européennes. Dans son mémoire rédigé en 1800, Péron s'appuie ainsi sur les travaux menés par les médecins Corvisart, Leroux et Pinel pour mettre au jour les relations entre les modes de vie souvent dégradés dans les «cités trop populeuses » et l'essor des maladies de « l'estomac et du cœur» (maladies cardio-vasculaires) et les «maladies nerveuses » (stress) qui seraient « devenues presque générales dans les grandes cités », en particulier à Paris. À l'inverse, il souligne que toutes les populations caractérisées par leur « défaut de civilisation » (ainsi « cette race d'hommes hideux et dégénérés qui végètent au milieu des glaces de Laponie », « l'habitant brûlé du désert », « l'apathique habitant des Antilles, et cette race immonde qui peuple la pointe méridionale de l'Afrique ») paraissent être dotées d'une "santé inaltérable ». C'est donc bien la critique des effets produits par la civilisation sur la dégradation de l'environnement (urbanisation, industrialisation, pollution) qui justifie, aux yeux de Péron, une certaine idéalisation de la vie sauvage, assimilée, à l'inverse, à une forme de protection de la nature et d'harmonie entre l'homme et son milieu.

En 1800, ces prises de position semblent constituer une résistance dans un contexte marqué par une «offensive industrielle $»^{28}$. À partir de 1795, l'émergence progressive d'une nouvelle forme de pouvoir technicoadministratif tend à bouleverser en profondeur les normes de régulation héritées du XVIII ${ }^{\mathrm{e}}$ siècle. Au nom du progrès, une partie de l'élite scientifique, cumulant le plus souvent des positions au sein des instances administratives et intellectuelles, participe alors à la légitimation d'innovations techniques ou industrielles dont les effets sur l'espace urbain et les modes de vie des populations sont présentés comme positifs. Les résistances à l'essor des industries polluantes et à la multiplication des accidents industriels

(28) Thomas Le ROUX, Le laboratoire des pollutions industrielles. Paris, 1770-1830, Paris, Albin Michel, L'évolution de l'humanité, 2011, p. 216-225 et p. 249. 
au cœur de Paris, existent au sein des populations ${ }^{29}$, résistances relayées par une partie de la communauté savante qui est loin d'être unanime face au « progrès ». Une même position critique est prise en 1800 par le jeune Virey qui critique l'usage des médicaments : il conviendrait, selon lui, de se tourner vers les peuples sauvages pour réapprendre l'usage de remèdes naturels et moins dangereux pour la santé. Loin d'être fondé sur une conception nostalgique ou idéalisée, le recours à la valorisation des populations naturelles et sauvages a ainsi pour objet de promouvoir un modèle alternatif et critique face aux discours tenus par une partie des élites scientifiques et administratives sur les bienfaits du progrès de la civilisation. Or, en 1807, qui tient ce discours critique contre le scientisme?

Dès 1800 , la critique du «progrès » devient la clef de voûte de l'offensive menée par les adversaires de la République directoriale auxquels le Consulat rend la parole. De Chateaubriand à Fontanes en passant par Ballanche et Bonald, la science et les savants deviennent les cibles privilégiées des nombreuses attaques qu'ils lancent pour dénoncer davantage le projet républicain du Directoire. Présentée comme «mortifère » et asséchante, «la » science est mise au pilori par une partie de cette élite intellectuelle, souvent royaliste et catholique, qui valorise à l'inverse les leçons de la nature contre la civilisation. La civilisation, symbolisée par la ville « monstrueuse », aurait, selon elle, provoqué une " dégénération » marquée, en particulier, par l'essor des suicides... et des divorces. À son retour en France, Péron se trouve face à cette offensive qui, prudente en 1800, s'est largement renforcée en 1807, grâce en particulier à la presse. Considérée dans cette perspective, son Voyage peut être lu comme une défense et illustration du rôle positif des sciences et des progrès de la civilisation : Péron prend position contre ceux qui valorisent la nature pour mettre en cause l'aspect positif des progrès scientifiques et techniques. Le Voyage de Péron peut être ainsi lu comme une réponse au... Génie du Christianisme et aux nombreuses attaques contre l'idée de civilisation héritée des Lumières :

"Que penser désormais de ces éloquentes déclamations contre le perfectionnement de l'ordre social, déduites de la force extraordinaire de l'homme sauvage, ou plutôt, pour parler le langage des sophistes, de l'homme de la nature !.... [...] Il me suffit d'avoir en ce genre ouvert la carrière de l'observation, et d'avoir opposé des expériences directes, des

(29) Jean-Baptiste FRESSOZ, L'apocalypse joyeuse, une histoire du risque technologique, Paris, Seuil, 2012. 
faits nombreux à cette opinion trop communément admise, trop dangereuse peut-être, et bien certainement trop exclusive, de la dégénération physique de l'homme par le perfectionnement de la civilisation $»^{30}$.

Répondant aux «poètes » et aux «philosophes » qui n'ont pas, comme lui, voyagé et fondé leurs observations des populations sur des mesures, Péron présente son récit comme une réponse aux « détracteurs de l'ordre social » qui prétendent que la civilisation serait synonyme de « dégradation ». Le fait que les peuples civilisés soient plus « forts » que les peuples sauvages prouve en effet le contraire. Péron devient, non seulement le porte-parole des défenseurs de la science, mais également de tous les « modernes » qui refusent de dénoncer les méfaits des progrès et d'idéaliser le passé et « la » nature.

Si la publication en 1807 de son Voyage peut constituer une réponse aux critiques portées contre les effets positifs des progrès de la civilisation, elle permet encore à Péron d'intervenir dans les débats sur les effets de la civilisation hors des territoires européens. Dès son retour en 1804, il intervient en effet dans ce débat par le biais d'un mémoire - encore trop méconnu par l'historiographie - qu'il remet au Conseiller d'État et membre de l'Institut, Antoine-François Fourcroy ${ }^{31}$. Sous le titre de Mémoire Sur les établissements anglais à la Nouvelle-Hollande, à la terre de Diémen et dans les archipels du grand Océan Pacifique, il propose déjà une description particulièrement riche des territoires parcourus, de leurs ressources naturelles et de leurs populations. Si ce mémoire a pu être lu comme le témoignage d'un projet d'expansion française aux antipodes $^{32}$, il peut également être compris comme une réponse à la question majeure que se posent alors des autorités françaises en butte à la déclaration d'indépendance d'Haïti, et plus généralement, aux mouvements de contestation qui se multiplient dans les diverses colonies : comment donc conserver des colonies et maintenir des populations sous tutelle $?^{33}$. On

(30) François PÉRON, Voyage, op. cit., p. 471.

(31) Ce mémoire (conservé dans le département des manuscrits de la BNF) a fait l'objet d'une édition critique publiée en 1998 par Roger Martin dans la Revue de l'Institut Napoléon : François PÉRON, « Mémoire sur les établissements anglais à la Nouvelle Hollande, à la Terre de Diémen et dans les archipels du grand océan Pacifique », Revue de l'institut Napoléon, 1998 - I, n ${ }^{\circ} 176$. Je tiens à remercier vivement John West-Sooby qui m'a permis de consulter sa propre transcription de ce mémoire conservé dans le département des manuscrits de la Bibliothèque nationale de France. Les citations sont issues de cette transcription manuscrite.

(32) Michael CONNOR, «The secret plan to invade Sydney », http://www.quadrant.org.au/ magazine/issue/2009/11/the-secret-plan-to-invade-sydney.

(33) Yves BÉNOT et Marcel DORIGNY (dir.), Rétablissement de l'esclavage dans les colonies françaises. Aux origines de Hä̈ti, Paris, Maisonneuve et Larose, 2003. 
comprend que l'objet principal des observations présentées dans ce mémoire soit moins les populations sauvages et indigènes que les populations européennes «transplantées » dans ces territoires situés aux antipodes. Comment ces populations se sont-elles adaptées ? Comment surtout sontelles parvenues à un haut degré de civilisation ? Ce sont bien là les deux questions principales auxquelles Péron tente de répondre.

Contrairement aux « préjugés » ou aux critiques dont elles peuvent être l'objet, Péron affirme que les récentes implantations anglaises (à Botany-Bay, Port-Jackson - futur Sydney- Paramatta...) sont parvenues à un «degré de splendeur et de prospérité qu'on ne savait pas même soupçonner en Europe et surtout en France $»^{34}$. Ce constat peut paraître surprenant tant la création de ces colonies est récente et surtout, tant la condition même des populations européennes ne pouvait laisser espérer de tels progrès. Colonies pénitentiaires, ces colonies sont pourtant présentées par Péron comme de véritables laboratoires de civilisation ${ }^{35}$. C'est en effet avec un enthousiasme souvent naïf qu'il décrit, au fil des rencontres, la société coloniale, véritable Arcadie des antipodes. Ce mémoire peut ainsi s'inscrire dans un corpus plus large constitué par les nombreux mémoires, statistiques et rapports envoyés par les différents agents français aux autorités administratives sous le Directoire et le Consulat, corpus réunissant les matériaux d'une « anthropologie diplomatique ${ }^{36}$. Après avoir dénoncé

(34) « Mémoire sur les établissements anglais à la Nouvelle-Hollande... », op. cit., chapitre 1, p. 1. Rappelons que ces nouvelles colonies datent de la décision prise par Georges III d'envoyer des criminels (convicts) hors du territoire britannique. À l'issue d'un voyage de huit mois, les onze bateaux composant l'expédition (732 convicts et 563 marins) accoste, en janvier 1788, sur les côtes de l'Australie à Botany Bay ainsi nommé par Cook. Les colons s'installent dans la baie Port Jackson où ils vivent pendant près de deux ans dans des conditions particulièrement difficiles.

(35) Péron n'est pas le premier à décrire la société coloniale de Botany Bay et de Port Jackson. Les récits des membres de la First Fleet rédigés par des marins anglais ont en effet circulé et on fait l'objet de traductions rapides dans toute l'Europe (particulièrement en Allemagne). En France, un des plus célèbres est celui de Watkin TENCH qui donne lieu à deux traductions en français en 1789, Voyage à la Baie Botanique ; avec une description du nouveau Pays de Galles Méridional, de ses habitants, de ses productions et quelques détails relatifs à M. de La Peyrouse, à Paris, Letellier, 1789 ; Relation d'une expédition à la Baie Botanique située dans la Nouvelle Hollande, sur la côté méridionale, nommée par le capitaine Cook, Nouvelles Galles méridionale, Paris, chez Knapen fils, 1789 (traduction de Ch. Pougens). Ce récit a fait l'objet d'une réédition récente : Expédition à Botany Bay. La fondation de l'Australie coloniale, introd. et notes d'Isabelle Merle, Toulouse, Anacharsis, 2006. D'autres récits suivront comme le Voyage du gouverneur Phillip à Botany Bay, avec une description de l'établissement des colonies du Port Jackson et de l'île de Norfolk, Paris, Buisson, 1791.

(36) Virginie MARTIN, « Du modèle à la pratique ou des pratiques aux modèles : la diplomatie républicaine du Directoire », dans Pierre SERNA (dir.), Républiques-sœurs. Le Directoire et la Révolution atlantique, Rennes, PUR, 2009, p. 87-100. On peut ainsi penser aux observations rapportées par André Thouin de sa mission en Belgique et aux Provinces-Unies entre 1794 et 1795. Cf. Ferdinand BoYER, « Le Muséum d'histoire naturelle à Paris et l'Europe des sciences sous la Convention », Revue d'histoire des sciences, 1973, 26, p. 251-257. 
les effets de l'isolement de la France provoqué par la Révolution, il se félicite des conditions nouvelles mises en place par le régime installé en 1799, ce dernier, favorisant les possibilités d'échanges et de commerce, doit désormais permettre à la civilisation de nouveaux progrès dont les colonies anglaises offrent justement le modèle. Il passe en revue les divers établissements anglais selon les modalités de description issues des enquêtes médicales et statistiques : aux conditions naturelles constituant le « milieu » (reliefs - rivières, montagnes -, climats, situation géographique...) succède la description des « richesses » et des différentes exploitations économiques (récoltes, plantations...). Il détaille ensuite les établissements installés au Diémen et en Nouvelle-Zélande, présentée comme le pivot du commerce anglais dans la région, et tous les établissements dans le Pacifique. Ce rappel des «particularités des conditions physiques du sol» lui permet de présenter les possibilités, mais aussi les contraintes, offertes à la colonisation anglaise $^{37}$.

Les descriptions se fixent principalement sur la colonie pénitentiaire de Port Jackson : il en décrit l'organisation politique, économique, sociale et culturelle à partir des rencontres avec des membres de la colonie, en particulier avec de nombreux Français, prisonniers, dont il raconte en détail les trajectoires. Contrairement aux allégations françaises, l'entreprise coloniale aurait permis d'améliorer, tant sur le plan physique que moral, les conditions des individus, les colonies devant dès lors constituer des modèles pour les métropoles. Preuve de ces effets positifs, Péron constate, par exemple, un accroissement démographique constant au sein des différentes sociétés européennes implantées dans les colonies. Il montre encore que ces sociétés, peuplées à l'origine de marginaux ou de brigands, sont désormais composées d'hommes et de femmes « civilisés ». La sécurité de la colonie, l'absence (apparente) de brigandage... sont autant de témoignages du succès de l'entreprise de colonisation. Il s'agit à chaque fois de mettre au jour les conditions favorables aux progrès et à l'accroissement rapide de ces colonies. L'auteur insiste moins sur les facteurs naturels que sur le rôle des institutions politiques et administratives qui permettent, à des degrés divers, la « régénération » des populations. Il accorde une attention particulière aux « criminels » et aux conditions de leur rééducation ${ }^{38}$. Pour

(37) Sur ce mémoire, voir l'étude particulièrement minutieuse de Margaret SANKEY, « The Baudin Expedition in Port Jackson, 1802 : Cultural Encounters and Enlightenment Politics », Explorations, 31, December 2001, p. 5-36.

(38) Id., « François-Auguste Péron : le mythe de l'homme sauvage et l'écriture de la science », Cahiers de sociologie économique et culturelle, 1988, 9, p. 37-46. 
les anciens prisonniers (en particulier les Français, voleurs, agioteurs, escrocs, aventuriers...), Port Jackson apparaît ainsi comme un cadre de rédemption. Selon Péron, le gouvernement anglais est parvenu à ce résultat grâce à deux instruments, la «terreur des lois » et les possibilités de perfectionnement (par le travail...).

Il analyse ainsi en détail les moyens nécessaires pour « corriger» et faire accéder au statut d'hommes libres les «serviteurs sous contrat» et les condamnés déportés dans ces colonies. Une des conditions du succès de l'entreprise coloniale renvoie au rôle joué par le gouvernement qui dirige l'entreprise puis s'efface pour laisser libres les initiatives privées. Les colons reçoivent ainsi l'aide des autorités qui pendant plusieurs années fournissent les moyens (alimentation, outils, mais aussi les femmes nécessaires aux mariages...) nécessaires à l'expansion de l'exploitation. Au bout d'un certain temps, les particuliers peuvent ainsi rembourser les prêts accordés par le gouvernement qui leur lègue les terres en pleine propriété en échange d'une redevance. Tout en équipant les colonies d'établissements nécessaires à l'éducation et à la santé, le gouvernement assure encore la défense des propriétés contre les brigands et favorise la fluidité sociale au sein des sociétés coloniales en permettant aux « criminels » repentis d'accéder à la communauté des hommes libres. L'obéissance aux lois et le travail permettent ainsi de renouveler en profondeur les sociétés. Ces observations sur la « régénération » opérée dans les colonies anglaises ne sont pas totalement neuves. En effet, dès 1789, dans la préface de la Relation d'une expédition à La Botanique de Watkin Tench qu'il traduit en français, Charles Pougens inscrit ce récit de voyage dans les débats qui ont alors lieu au sein de l'Assemblée constituante sur la réforme de la justice : faut-il enfermer ou exiler les criminels ? L'expérience de «transportation » outre-mer menée par les Anglais ${ }^{39}$ suscite encore des

(39) Les Anglais ont longtemps envoyé leurs prisonniers indésirables dans les colonies américaines jusqu'en 1783. L'Australie devient sur les conseils du naturaliste et secrétaire de la Royal Society, Joseph Banks, la destination vers laquelle il convient ensuite d'envoyer les prisonniers. Cette expérience suscite l'intérêt de Charles Pougens : «L'idée de tirer parti d'un pays, en y établissant des colonies agricoles, n'est pas nouvelle; mais ce sont les moyens employés pour la faire réussir que nous osons présenter ici comme nouveaux : tels sont le choix du lieu, les soins que les Anglais prennent de ces hommes coupables; soins qui n'ont pas seulement pour principe cette sensibilité physique que tant de circonstances peuvent faire varier et même errer ; mais cette sensibilité philosophique dont l'impression est plus durable, parce qu'elle est fondée sur un calcul, dont les éléments sont le bonheur de chaque individu, et celui de la société. Nous osons donc espérer qu'un si bel exemple sera suivi par les Français, qui ne le cèdent à aucun peuple en sensibilité et en humanité », dans Watkin TENCH, Relation d'une expédition à la Baie Botanique située dans la Nouvelle Hollande, sur la côte méridionale, nommée par le capitaine Cook, Nouvelles 
débats sous le Directoire et l'Empire ${ }^{40}$.

Une autre raison du succès de la colonisation vient du fait que le gouvernement a en quelque sorte encouragé la mixité des populations : aux côtés des « criminels », le gouvernement anglais a en effet déporté des prisonniers politiques (Irlandais en particulier) qui, souvent issus des élites sociales, sont venus progressivement se mélanger avec les catégories les plus « basses ». Dès lors, une colonie comme Botany-Bay est devenue attractive, même pour les familles « libres et honnêtes qui viennent profiter des bienfaits du gouvernement et des avantages du climat et du sol ». Pour Péron, la réussite de l'entreprise de colonisation s'explique justement parce qu'elle s'appuie sur des populations marginales ( «En effet si l'on observe que les filles publiques appartiennent dans tous les pays à la partie la plus robuste et la plus belle de la population; si l'on observe qu'un tel état et les chances diverses qu'il comporte a pour résultat nécessaire de donner une sorte d'exaltation aux idées ; qu'à plus forte raison encore l'état de brigand sur les routes publiques, celui de voleur dans les grandes cités exigent de la force, de l'adresse et une sorte d'audace qui n'est pas l'apanage du commun des hommes, il doit en résulter que soit pour le physique soit pour l'intelligence les enfants de tels époux n'ont pas été aussi mal partagés qu'on pourrait le croire $\gg^{41}$ ). La création de maisons d'éducation, le maintien d'une force coercitive permettent ainsi au gouvernement anglais de créer les conditions favorables à l'expansion économique et à l'équilibre social.

Observant de manière positive les colonies pénitentiaires anglaises, Péron insiste sur le fait qu'il serait donc inutile de vouloir s'emparer militairement de ces colonies si le gouvernement français n'est pas prêt à maintenir les conditions qui ont permis le succès de ce processus : «Or

Galles méridionale, Paris, chez Knapen fils, 1789, Avertissement du traducteur. Notons que Charles Pougens traduira encore sous le Directoire un ouvrage consacré aux colonies anglaises d'Australie : Voyage à la Nouvelle Galles du Sud, à Botany-bay, au Port Jackson, en 1787, 1788, 1789, par John White,... Ouvrage où l'on trouve de nouveaux détails sur le caractère et les usages des habitants du cap de Bonne-Espérance, de l'île Ténériffe, de Rio Janeiro et de la Nouvelle-Hollande, ainsi qu'une description exacte de plusieurs animaux inconnus jusqu'à présent. Traduit de l'anglais avec des notes critiques et philosophiques..., Paris, Guillaume, An VI/1798. On sait que la France met en place des colonies pénitentiaires dans le second XIX ${ }^{\mathrm{e}}$ siècle, en Nouvelle-Calédonie : Isabelle MERLE, Expériences coloniales. La Nouvelle-Calédonie. 1853-1920, Paris, Belon, 1995.

(40) Je remercie Bernard Gainot des éclaircissements concernant les débats autour de la publication de l'ouvrage de Charles-Antoine-Joseph Leclerc de MonTLINOT, Essai sur la transportation comme récompense, et la déportation comme peine, Paris, impr. de Gratiot, an V/1796. (41) « Mémoire sur les établissements anglais à la Nouvelle-Hollande... », op. cit., chap. 2, p. 8 . 
dans la supposition d'une entreprise contre ces colonies ne devient-il pas indispensable que vous connaissiez davantage tous les principes de l'organisation de ces colonies afin de consacrer dans vos instructions ceux qui vous paraîtront devoir être conservés et de modifier ceux qui vous en paraîtraient susceptibles $»^{42}$. Précisément, selon lui, la mise en place de l'esclavage constituerait une erreur. En continuité avec les principes de la « colonisation nouvelle » défendue sous le Directoire, Péron affirme en effet que le succès de l'entreprise coloniale menée par les Anglais s'explique justement par le fait de ne pas avoir tenté de réduire les populations locales à l'esclavage ${ }^{43}$. Le fait qu'il soustrait au travail les maîtres provoque une dégénération chez ces derniers liée à leur inactivité :

«Les colons anglais vivent avec ces sauvages en fort bonne intelligence; ils ne cherchent point à les asservir, leur laissent toute liberté de suivre leurs goûts et leurs habitudes ; rarement ont-ils été obligés de se défendre de leurs coups, même dans les premiers temps qu'ils sont venus habiter sur ces bords. [...] Quoique ces peuples soient naturellement ennemis du travail, on a lieu d'espérer qu'avec des soins et de la patience, on parviendra à les y porter ; quelques colons en ont fait l'expérience et ont obtenu d'heureux résultats ; il est vrai qu'ils avaient pris ces sauvages fort jeunes chez eux, mais de leur plein gré et sans employer la force ni la violence $»^{44}$.

Dans ce mémoire, Péron propose ainsi un véritable «programme » de gouvernement colonial qui, on le sait, ne sera pas suivi par le pouvoir napoléonien occupé principalement à une domination sur le continent européen ${ }^{45}$. Péron semble ainsi réduire les possibilités de «progrès » et d'amélioration des populations et des sociétés aux seuls groupes européens. Dans ce mémoire, il ne se désintéresse pas totalement des populations sauvages qu'il présente souvent comme victimes de la conquête anglaise ${ }^{46}$. Reste que ces populations apparaissent dans ce mémoire comme « exclues », ou du moins à l'écart du processus de civilisation.

(42) Ibidem, p. 1.

(43) Bernard GAINOT, « La Décade et la "colonisation nouvelle" », AHRF, n³39, 2005, p. $99-116$.

(44) François PÉRON, Voyage..., op. cit., p. 294.

(45) Massimiliano VAGHI, La relation du siège de Pondichéry (1748) en forme de journal. Un episodio chiave della rivalità anglo-francese in India, Milan, CUEM, 2010, p. 32-35 et p. 79-102 ; $i d$., « L'Europa in Oriente. La crisi delle potenze asiatiche e le origini dell' imperialismo europeo », Quaderni di Relazioni Internazionali, num. 6 (dicembre 2007), ISPI, Milano, p. 68-82.

(46) « Mémoire sur les établissements anglais à la Nouvelle-Hollande... », op. cit., chapitre 1, p. 11. 
Le regard européocentriste, les préjugés exprimés contre les populations constituent sans aucun doute une rupture avec les principes des Lumières repris, en partie, dans le projet anthropologique des Observateurs de l'homme. Mais, contrairement à d'autres théoriciens esclavagistes et racistes (Malouet, Barré de Saint-Venant), Péron ne « fixe» ni ne naturalise pas complètement l'infériorité des populations aborigènes ${ }^{47}$, justifiant d'ailleurs son souci réel de communiquer avec elles et de les comprendre ${ }^{48}$. Il se contente de renvoyer à un futur, lointain et utopique, les possibilités même de progrès des populations sauvages. Mais de ce fait, et c'est en cela qu'il invente le $\mathrm{XIX}^{\mathrm{e}}$ siècle et rompt avec l'histoire naturelle de Buffon, Péron contribue à scinder en deux l'histoire de l'humanité : d'un côté, il place les «forts », les civilisés, ceux qui ont une histoire et dont il est possible d'écrire l'histoire individuelle et collective ; de l'autre, les «faibles », les sauvages, qui vivent dans une espèce d'invariant temporel, ceux qui n'ont pas d'histoire. Cette partition rend dès lors légitime une entreprise de colonisation qui consiste à exporter la civilisation hors des territoires européens et donc de faire entrer dans l'histoire des territoires et des populations qui n'en auraient pas. Cette coupure constitue bien une étape majeure qui est progressivement formalisée et s'impose comme une véritable doxa au $\mathrm{XIX}^{\mathrm{e}}$ siècle ${ }^{49}$. Que Péron soit redécouvert à la fin du $\mathrm{XIX}^{\mathrm{e}}$ siècle est sans doute tout autant le fait de théoriciens qui entendent légitimer la hiérarchie des races sur des mesures physiques, que de penseurs de la III ${ }^{\mathrm{e}}$ République qui entendent justifier une entreprise coloniale fondée désormais sur l'idée qu'entre peuples civilisés et peuples sauvages, il ne saurait plus y avoir, contrairement à l'idée défendue sous le Directoire et

(47) Constatant, dans le récit du voyage, la proximité physique entre les corps, constatant encore la possibilité d'échanges avec les indigènes, Péron met en cause la « supériorité » de la couleur blanche et affirme une sorte de relativisme.

(48) Stephanie ANDERSON, « French Anthropology in Australia : The First Fiekdwork Report : François Péron's 'Maria Island : Anthropological Observations », Aborigical History, 2001, vol. 25

(49) Voir Eusèbe de SALVERTE qui établit une distinction entre « deux formes de civilisation essentiellement différentes, la forme fixe et la forme perfectible : la première, commune à un grand nombre de peuples anciens, subsiste encore au Tibet et à la Chine ; la seconde est celle qui, depuis plusieurs siècles, suit en Europe sa marche progressive ; celle qui a conquis l'Amérique septentrionale et qui ouvre à l'Amérique méridionale le chemin de l'instruction et de la prospérité », Essai historique et philosophique sur les noms d'hommes, de peuples et de lieux, Paris, impr. de Lachevardière, 1824, t. 1, p. ii. 
reprise par Gérando dans ses « Considérations », une co-temporalité, c'est à dire une histoire commune et partagée ${ }^{50}$.

Jean-Luc CHAPPEY

Université de Paris 1 Panthéon-Sorbonne

IHMC/IHRF

jlchappey@gmail.com 